\title{
Scaling of Nanoparticle Retardation in Semi-dilute Polymer Solutions
}

\author{
Remco Tuinier ${ }^{1}$ and Tai-Hsi Fan ${ }^{2}$ \\ ${ }^{1}$ Helmholtz Zentrum Jülich, Institut für Festkörperforschung, Soft Matter, 52425 Jülich, Germany \\ ${ }^{2}$ Department of Mechanical Engineering, University of Connecticut, Storrs, CT 06269-3139, USA
}

(Dated: October 29, 2007)

\begin{abstract}
We analyze the scaling law for the polymer-induced retardation a nanoparticle experiences as it moves through a semi-dilute polymer solution. The translational friction is calculated from a modified Stokes flow using a local viscosity near the nanosphere. The results rationalize a general retardation factor, $R=\exp \left(K a^{\mu} c^{\nu}\right)$ [T. Odijk, Biophys. J. 79 (2000) 2314], revealing scaling exponents $\mu=0.77$ and $\nu=1$, which are in agreement with experiment. We find that rotational motion also has a self-similar behavior and $R$ can be described too by a stretched exponential with slightly different exponents.
\end{abstract}

\section{INTRODUCTION}

Nanoparticle transport through media crowded with macromolecules is of interest in (cell) biology [1, 2], colloid science [3-6], and soft condensed matter physics [712]. Understanding nanoscale motion such as the diffusion of globular proteins through polymer solutions may reveal diffusion-limited biochemical reactions within a crowded biological cell [13]. Transport behavior such as particle mobility is often used as a tool to characterize proteins using e.g. electrophoresis, photon or fluorescence correlation spectroscopy [1]. Experimentally, methods including sedimentation [4], diffusion [3] and electrophoresis [14] of dilute nanoparticles in a polymer solution yield a transport quantity $Q$ that is commonly compared with its value $Q_{0}$ in absence of polymer.

The decrease of $Q$ with respect to $Q_{0}$ upon adding polymer to the solution is widely described in terms of a so-called retardation factor $R$. The retardation factor is a dimensionless number between unity (no retardation) and infinity (full retardation) that describes the slowing down of particle motion as affected by added polymers. In many experimental studies, e.g. [3, 14, 15], semi-empirical stretched exponential functions are used to describe the concentration-, nanosphere size-, and molar mass-dependence on the relative transport quantities $Q / Q_{0}$. Odijk [16] suggested, on the basis of scaling laws [17-19] and an extended set of experimental data, the following general stretched exponential form for the retardation factor $R$ as a function of the nanosphere radius $a$ and polymer bulk concentration $c_{b}$ :

$$
R=\exp \left(K a^{\mu} c_{b}{ }^{\nu}\right)
$$

or in dimensionless form

$$
\ln R=K^{\prime}\left(\frac{a}{\delta}\right)^{\omega}\left(c_{b}[\eta]\right)^{\chi},
$$

where $K^{\prime}$ is the (dimensionless) retardation coefficient, $\delta$ is the characteristic polymer depletion thickness and $[\eta]$ is the intrinsic viscosity of the polymer solution, of which the inverse is close to $c_{b}{ }^{*}$, the polymer overlap concentration. A few hypotheses were proposed to rationalize the scaling exponents, however, there is yet no satisfactory theoretical proof that $R$ can be expressed in such a general fashion. From several sets of experimental data it follows the retardation scaling behavior reduces to 0.69 $\leq \mu \leq 1$ and $0.5 \leq \nu \leq 1.1$ [16].

In this Communication we perform a fluid flow analysis and compute the retardation factor based on polymer depletion theory. It is assumed structural relaxations in the polymer solution [20, 21] near the particle surface are fast compared to the velocity time scale of the moving nanoparticle, implying the depletion thickness is a constant during sphere motion. This is a fair approximation if the chains are smaller than the sphere. Odijk [16], however, found the convective effect is negligible also for small radii. Therefore our model could be accurate for a wide range of polymer-to-sphere size ratios. We show that eq. 1 holds generally in the semi-dilute polymer concentration regime for translational and rotational motion. We consider a nanosphere in a semi-dilute polymer solution with nonadsorbing chains. Around such a sphere one expects a layer depleted of polymer segments $[20,22,23]$. Depletion of polymer chains induces interesting phase behavior in colloid-polymer mixtures [24-28], enabling visualization of wetting phenomena $[29,30]$ and even capillary waves [31] at the particle level. The depletion of polymer segments next to a nonadsorbing surface is due to the loss of configurational entropy of a chain near a surface. 


\section{THEORY}

We assume the local viscosity follows the polymer segment density profile $c(z)$ around the sphere [32]. For the relative density profile $\rho(z)=c(z) / c_{b}$ of polymer segments, as a function of the radial distance $z$ from the sphere surface, we use the general form $\rho(z)=$ $[z / a+\tanh (z / \delta)]^{2} /[z / a+1]^{2}[33]$. The depletion thickness $\delta$ is close to the polymer's radius of gyration in dilute solution [34] and decreases with concentration for $c_{b}>c_{b}{ }^{*}$ $[20,33]$. The convective effect is negligible for small radii [16], so it is assumed the local viscosity $\eta(z)$ follows the equilibrium density profile [32, 35], expressed as

$$
\eta(z) / \eta_{0}=1+[\eta] c_{b} \rho(z) e^{k_{H}[\eta] c_{b} \rho(z)},
$$

which is a modified version [32] of the semi-empirical Martin equation [36-38] for the local viscosity near a nanosphere. Essentially, we can account for any concentration-dependent viscosity, and here only the Martin equation is applied as a representative case. In eq. $2 \eta_{0}$ is the solvent viscosity and $k_{H}$ is the Huggins coefficient, which we set here at a realistic value of 0.5 . The Martin equation for $\eta_{p}$, the viscosity of the polymer solution in the bulk, $\eta_{p} / \eta_{0}=1+[\eta] c_{b} \exp \left(k_{H}[\eta] c_{b}\right)$, describes the relative viscosity of polymer solutions up to high polymer concentrations. In the dilute concentration regime the Martin equation is consistent with the Huggins equation.

To calculate the hydrodynamic resistance the nanoparticle experience, we use the linearized equation of motion for low Reynolds number flow with local viscosity effect:

$$
0=-\nabla p+\eta \nabla^{2} \mathbf{v}+\nabla \eta \cdot\left[\nabla \mathbf{v}+(\nabla \mathbf{v})^{T}\right]
$$

where $\eta$ is the local viscosity that depends on local concentration $c(\mathbf{r}), p$ is the pressure field, $\mathbf{v}$ is the velocity, and $\nabla \mathbf{v}+(\nabla \mathbf{v})^{T}$ is the strain rate tensor. For a spherical particle, the fluid flow problem introduced by the translational motion of the particle can be simplified by using the Stokes stream function [39, 40], which we extended in order to account for a local viscosity profile. After replacing the velocity components by the Stokes stream function $\varphi$ and applying the trial solution $\varphi=\sin ^{2}(\theta) f(r)$, a $4^{\text {th }}$-order differential equation for the radial function $f(r)$ is obtained [41]:

$$
\begin{aligned}
& 0=f^{(4)}+\frac{2 \eta^{\prime}}{\eta} f^{\prime \prime \prime}-\left(\frac{4}{r^{2}}+\frac{2 \eta^{\prime}}{r \eta}-\frac{\eta^{\prime \prime}}{\eta}\right) f^{\prime \prime} \\
& +\left(\frac{8}{r^{3}}-\frac{2 \eta^{\prime}}{r^{2} \eta}-\frac{2 \eta^{\prime \prime}}{r \eta}\right) f^{\prime}-\left(\frac{8}{r^{4}}-\frac{8 \eta^{\prime}}{r^{3} \eta}-\frac{2 \eta^{\prime \prime}}{r^{2} \eta}\right) f
\end{aligned}
$$

with no-slip and vanishing far-field boundary conditions. From analytical integration of the momentum equation, we find that the corresponding pressure field at the particle surface is $p(1, \theta)=-\cos (\theta) f^{\prime \prime \prime}(1)$, the normal stress vanishes, and the surface shear stress becomes $\sigma_{1, \theta}(1, \theta)=\sin (\theta)\left[1+f^{\prime \prime}(1)\right]$. After integration of the surface traction we obtain an expression for the translational frictional coefficient, which can be expressed as $6 \pi \eta_{0} g^{t} a$, including the correction function $g^{t}$ to the Stokes friction $6 \pi \eta_{0} a$ :

$$
g^{t}=\frac{\eta_{\mathrm{eff}}}{\eta_{0}}=\frac{4}{9}+\frac{4}{9} f^{\prime \prime}(1)-\frac{2}{9} f^{\prime \prime \prime}(1) .
$$

Equation (4) was solved numerically yielding the ingredients for the calculation of $g^{t}$ using (5). It is noted we can solve eq. 4 and compute the effective viscosity with eq. 5 for any density profile, hence viscosity profile. This implies our theory can be applied to several other situations as well. For instance, computing the effective viscosity experienced by a colloid with anchored brushes as they diffuse through a solvent.

Next we need to relate this correction function $g^{t}$ to the retardation factor $R$. The physical definition of $R$ is similar to $g^{t}$, which equals the viscosity ratio $\eta_{\mathrm{eff}} / \eta_{0}$, measured through $Q_{0} / Q$. In case of self-diffusion measurements for instance this ratio equals the relative diffusivity $D_{0} / D_{\text {eff. }}$ The retardation factor $R$ is however not equal to $g^{t}$; the stretched exponential form $R=\exp \left[K a^{\mu} c^{\nu}\right]$ has an upper bound $R=\infty$ that differs from the limiting upper value $\eta_{p} / \eta_{0}$ for $g^{t}$. Neither does $R=1$ provide the correct lower bound, because it follows from our numerical results $g^{t}$ does not approach unity in the protein limit due to the local viscous effect described by the corresponding density profile. To match $g^{t}$ from numerical calculation with the retardation factor $R$, we need to rescale the stretched exponential form spanning over the full range of possible viscosity ratios as

$$
\frac{Q}{Q_{0}}=\frac{1}{g^{t}}=\frac{1}{R}(\kappa-\lambda)+\lambda,
$$

where the corrected upper and lower limits for $g^{t}$ are

$$
\kappa=\lim _{a \rightarrow 0} \frac{1}{g^{t}} \quad \text { and } \quad \lambda=\lim _{a \rightarrow \infty} \frac{1}{g^{t}}=\frac{\eta_{0}}{\eta_{p}},
$$

respectively. Therefore, the retardation factor $R$ is connected with the numerical model through $R=(\kappa-$ $\lambda) /\left(1 / g^{t}-\lambda\right)$. We explain these limiting forms as follows. As a spherical particle moves through a polymer solution it is retarded in its motion as compared to 'free' diffusion in a pure solvent. In the 'colloid limit', where the sphere is large $(a \rightarrow \infty)$ compared to any length scale (the depletion thickness vanishes), $1 / g^{t}$ equals $\lambda$, so the friction coefficient equals $6 \pi \eta_{p} a$, with $\eta_{p}$ the viscosity of the polymer solution. This is the maximum frictional force that can be reached and the retardation factor $R$ becomes infinitely large. In the so-called 'protein limit' the retardation is weakest; the depletion layer is then rather extended compared to the sphere size and becomes independent of the characteristic polymer length scale. We define this limit as non-retarded; $1 / g^{t} \rightarrow \kappa$ and $R \rightarrow 1$. In that limit of a translating small sphere, the relative density profile $\rho(z)$ of polymer segments attains $\rho(z)=[z /(z+a)]^{2}[42]$. Using this simplified form we find that the first-order asymptote for $g^{t}$ in the dilute 
limit is $g^{t}=1+(89 / 210)[\eta] c_{b}[41]$. Beyond the overlap concentration we analyzed $\kappa$ numerically and find a simple dependence of $\kappa^{-1}$ on $c_{b}[\eta]$, see the inset in Fig. 1.

\section{RESULTS}

The scaling law for the particle retardation effect can now be analyzed. The relative effective friction coefficient $g^{t}$ was computed for a broad range of polymer concentrations in the semi-dilute regime. We then performed a global fitting to all data using eq. 1 and found scaling exponents $\omega=0.77$ and $\chi=0.44$ and a retardation coefficient $K^{\prime}=0.69$. For polymer concentrations from $[\eta] c_{b}=2$ up to 10 in the semidilute regime, that has an onset at $c_{b}>[\eta]$, we plot $1 / g^{t}$ as a function of $a / \delta$, see Fig. 1 . We note that adjusting the Huggins coefficient only affects the value for $K^{\prime}$. Because the depletion thickness $\delta$ is close to the correlation length in a polymer solution [20], $\delta$ scales as $c_{b}^{-3 / 4}$ in the semi-dilute regime in a good solvent, so we find exponents $\mu=0.77$ and $\nu \approx 1.0$. This is consistent with experimental observations (see Table 1 in ref. [16]). As an illustration, for $[\eta] c_{b}=2$ and 3 , we added the analytical result for the two layer approach [35], which is exact in the colloid limit, but obviously deviates for $a<2 \delta$.

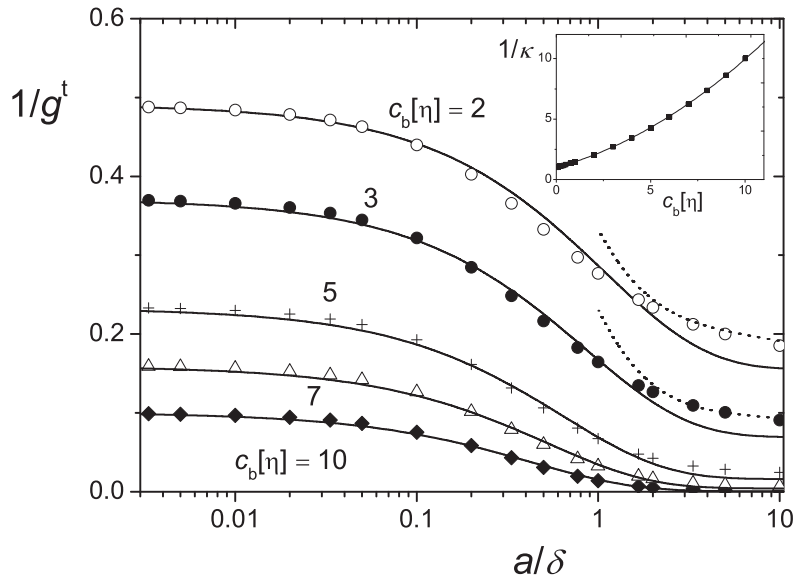

FIG. 1: Normalized friction coefficient $g^{t}$ for translational motion as a function of the sphere radius $a$ scaled by depletion thickness $\delta$ for five polymer concentrations indicated. Data: numerical results. Solid curves follow eq. 6 with $\ln R=0.69(a / \delta)^{0.77}\left(c_{b}[\eta]\right)^{0.44}$. The retardation coefficient 0.69 and the two stretching exponents are obtained by simultaneously fitting all numerical data. The dashed curve is the result using the two-layer model [35]. Inset: $\kappa$ as a function of the polymer concentration. Data points: numerical solutions; solid curve follows $g^{t}=1+(89 / 210)[\eta] c_{b}+0.047\left([\eta] c_{b}\right)^{2}$.

In Fig. 2 we plotted the numerical values obtained for the retardation factor $R$ versus $0.69(a / \delta)^{0.77}\left([\eta] c_{b}\right)^{0.44}$ and find all data collapse onto a single curve. Both for small and large $a$ we do find deviations but especially when $a \geq \delta$ the general stretched exponential retardation factor works very well. Let us estimate the relevant size of $a / \delta$ for translational diffusion of a protein.

If we take a globular protein with a radius of $2 \mathrm{~nm}$ (e.g. lysozyme, BSA, $\beta$-lactoglobulin or $\alpha$-lactalbumin) that moves in a solution with polymer chains that have a radius of gyration $R_{g}$ of, say, $20 \mathrm{~nm}$, we may use again that $\delta / R_{g}$ scales as $\left(c_{b} / c_{b}^{*}\right)^{-3 / 4}$ in the semi-dilute regime in a good solvent [20]. For a typical value of $c_{b} / c_{b}^{*}$ between 2 and $10, a / \delta=0.1$ to 0.6 , and this is the regime where the stretched exponential function $R$ is in close agreement with the numerical data, shown in Figs. 1 and 2 (note that $K^{\prime}\left([\eta] c_{b}\right)^{0.44}$ is of the order of unity). The solid curve gives $\ln R=0.69(a / \delta)^{0.77}\left([\eta] c_{b}\right)^{0.44}$. In the inset $R^{-1}$ is plotted logarithmically, demonstrating the linear dependence of $\ln R$ on $(a / \delta)^{0.77}\left([\eta] c_{b}\right)^{0.44}$.

An important aspect that follows from this work is that the retardation factor for translational mobility is similar but not equal to $g^{t}$, which is determined experimentally using a normalized transport quantity $Q / Q_{0}$. In order to describe experimental results in terms of eq. 1 one needs eq. 6 including $\lambda$ and $\kappa$, the limiting inverse relative viscosities of the polymer solution a sphere experiences in the colloid and protein limits, respectively. At present, the interpretation of many experimental results is based on $R=\exp \left(Q_{0} / Q\right)$. Accounting for the limiting values is important in evaluating the stretched exponential scaling factors. Therefore, experimental data should be (re)analyzed as to verify the proposed theoretical scaling exponents. Possibly, the spread in the exponents from experimental data becomes smaller if our rescaling is used.

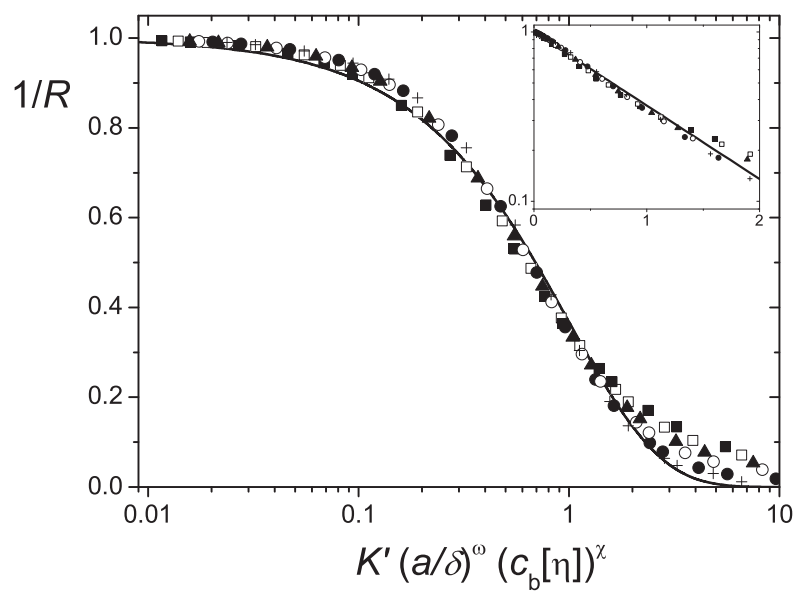

FIG. 2: Translational motion retardation factor $R$ as a function of $K^{\prime}(a / \delta)^{\omega}\left([\eta] c_{b}\right)^{\chi}$, with $\omega=0.77, \chi=0.44$ and $K^{\prime}=0.69$ for $[\eta] c_{b}=2,3,5,7$ and 10 (data points). The solid curve follows $R^{-1}=\exp \left(-0.69(a / \delta)^{0.77}\left([\eta] c_{b}\right)^{0.44}\right)$. Inset: Identical data and curve with $R^{-1}$ plotted logarithmically.

We find that the self-similar retardation effect on rotational motion can also be described by the stretched exponential retardation factor with slightly different ex- 
ponents. Based on eq. 3 we deduce a $2^{n d}$-order ODE:

$$
0=w^{\prime \prime}+\left(\frac{2}{r}+\frac{\eta^{\prime}}{\eta}\right) w^{\prime}-\left(\frac{2}{r^{2}}+\frac{\eta^{\prime}}{\eta r}\right) w
$$

for the radial function $w(r)$ that connects to the azimuthal velocity component. The corresponding no-slip and vanishing boundary conditions are $w(1)=1$ and $w(r \rightarrow \infty)=0$.

In order to quantify the rotational frictional coefficient, which can be expressed as $8 \pi \eta_{0} g^{r} a^{3}$, with the following result for the correction function $g^{r}$ :

$$
g^{r}=\frac{1}{3}\left[1-w^{\prime}(1)\right]
$$

where $w^{\prime}(1)$ is again solved numerically. For rotational motion we rescale $g^{r}$ through $R=(\iota-\lambda) /\left(1 / g^{r}-\lambda\right)$, with upper and lower limits for $g^{r}$ :

$$
\iota=\lim _{a \rightarrow 0} \frac{1}{g^{r}} \quad \text { and } \quad \lambda=\lim _{a \rightarrow \infty} \frac{1}{g^{r}}=\frac{\eta_{0}}{\eta_{p}},
$$

respectively. For the quantity $\iota$ we find an analytical expression in the dilute concentration regime, $\iota^{-1}=$ $\left.1+\left(c_{b}[\eta]\right) / 10\right)[41]$. In the semidilute regime the numerical data could be described accurately with $\iota^{-1}=$ $1+0.1334\left(c_{b}[\eta]\right)^{0.714}$. Simultaneous fitting the numerical $g^{r}$ results as a function of $a / \delta$ for various concentrations gave an accurate description of the data using eq. 1 with $\omega=0.67, \chi=0.16$ and $K^{\prime}=0.325$. The main difference with translational motion is the large difference of the retardation coefficient $K^{\prime}$. This smaller value tells that rotational motion is less retarded than translational motion.

This can be explained by the fact that, as a function of the distance from the sphere, the velocity field decays faster in case of rotation. A rotating nanosphere mainly senses the near-field viscosity where it is closer to the solvent viscosity, whereas a translating sphere senses relatively more of the far-field viscosity. The above data mean that the scaling exponents for rotation are equal to $\mu=0.67$ and $\nu \approx 0.66$ for good solvent conditions.

In Fig. 3 it is shown all numerical data points fall onto a single curve for rotational motion and the retardation factor is again described using the stretched exponential scaling law. It will be interesting to compare our results with experimental data on rotational friction, which are yet scarcely available.

\section{CONCLUDING REMARKS}

In conclusion we have shown the often proposed general semi-empirical stretched exponential function for the polymer-mediated retardation of nanoparticles in solutions with semi-dilute chains is accurate. We gave theoretical values for the retardation coefficient and scaling exponents for translational and rotational motion. It was shown rescaling the relative effective viscosity is essential

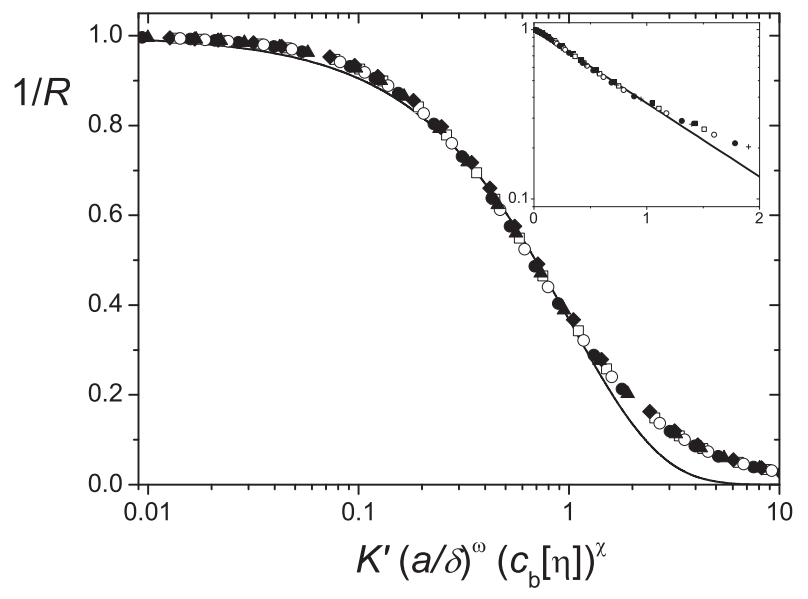

FIG. 3: Retardation factor $R$ for rotational motion as a function of $K^{\prime}(a / \delta)^{\omega}\left([\eta] c_{b}\right)^{\chi}$, with $\omega=0.67, \chi=0.16$ and $K^{\prime}=0.325$ for $[\eta] c_{b}=3,4,5,7$ and 10 (data points). The solid curve follows $R^{-1}=\exp \left(-0.325(a / \delta)^{0.67}\left([\eta] c_{b}\right)^{0.16}\right)$. Inset: Identical data and curve with $R^{-1}$ plotted logarithmically.

in order to connect with the retardation function that is used often in practice. The retardation coefficient is much smaller for rotational motion.

\section{Acknowledgment}

T.-H. Fan is grateful for the financial support of this work from the University of Connecticut Research Foundation. We thank D.G.A.L. Aarts and G. Nägele for useful remarks on the manuscript and J.K.G. Dhont for continuous support. 
[1] J. Lippincott-Schwartz et al. Nature Rev. Mol. Cell Biol., 2:444, 2001.

[2] R.J. Ellis and A.P. Minton. Nature, 425:27, 2003.

[3] G.S. Ullmann et al. J. Phys. Chem., 89:692, 1985.

[4] P. Tong et al. Phys. Rev. Lett., 79:2363, 1997.

[5] G.H. Koenderink et al. Phys. Rev. E, 69:021804, 2004.

[6] K. Kang et al. J. Chem. Phys., 122:044905, 2005.

[7] T. Gisler and D.A. Weitz. Curr. Opin. Colloid Interface Sci., 3:586, 1998.

[8] F. Brochard Wyart and P.G. de Gennes Eur. Phys. J. E, 1:93, 2000.

[9] J.C. Crocker et al. Phys. Rev. Lett., 85:888, 2000.

[10] T. Odijk Physica A, 389:337, 2004.

[11] T.A. Waigh. Rep. Progr. Phys., 68:685, 2005.

[12] J. Szymańiski et al. J. Phys. Chem. B, 25593:110, 2006.

[13] R.J. Ellis. Trends Biochem. Sci., 26:597, 2001.

[14] S.P. Radko and A. Chrambach. Biopolymers, 4:183, 1997.

[15] W. Brown and R. Rymdén. Macromolecules, 21:840, 1988.

[16] T. Odijk. Biophys. J., 79:2314, 2000.

[17] A.G. Ogston, B.N. Preston, and J.D. Wells. Proc. $R$. Soc. Lond. A., 333:297, 1973.

[18] D. Langevin and F. Rondelez. Polymer, 19:875, 1978.

[19] R.I. Cukier. Macromolecules, 17:252, 1984.

[20] P.G. de Gennes. Scaling concepts in polymer physics. Cornell University Press, Ithaca, 1979.

[21] M. Doi and S.F. Edwards. The Theory of Polymer Dynamics. Oxford Science Publishers, 1986.

[22] S. Asakura and F. Oosawa. J. Chem. Phys., 22:1255, 1954.
[23] A. Vrij. Pure Appl. Chem., 48:471, 1976.

[24] A.P. Gast et al. J. Colloid Interface Sci., 96:251, 1983.

[25] H.N.W. Lekkerkerker et al. Europhys. Lett., 20:559, 1992.

[26] M. Fuchs and K.S. Schweizer. Europhys. Lett., 51:621, 2000.

[27] P.G. Bolhuis et al. Phys. Rev. Lett., 89:128302, 2002.

[28] P.G. Bolhuis et al. Phys. Rev. Lett., 90:068304, 2003.

[29] D.G.A.L. Aarts et al. J. Phys: Condens. Matter, 15:S245, 2003.

[30] W.K. Wijting et al. Phys. Rev. Lett., 90:196101, 2003.

[31] D.G.A.L. Aarts et al. Science, 304:847, 2004.

[32] R. Tuinier and T. Taniguchi. J. Phys: Condens. Matter, 17:L9, 2005.

[33] G.J. Fleer et al. Macromolecules, 36:7857, 2003.

[34] E. Eisenriegler. J. Chem. Phys., 79:1052, 1983.

[35] R. Tuinier et al. Europhys. Lett., 75:929, 2006.

[36] A.F. Martin. American Chemical Society Meeting, Memphis, discussed by [37]:April 20-24, 1942.

[37] W. E. Davis and J. H. Elliott. Cellulose and Cellulose derivatives, p. III, 2nd ed., E. Ott, H. Spurlin and M.W. Grafflin, Eds. Interscience, New York, 1955.

[38] F. Rodriguez. Polym. Lett. Ed., 11:485, 1973.

[39] G.G. Stokes. Trans. Camb. Phil. Soc., 9:8-106, 1851.

[40] J. Happel and H. Brenner. Low Reynolds Number Hydrodynamics. Prentice-Hall, New York, 1965.

[41] T.-H. Fan, B. Xie, and R. Tuinier. unpublished work, to be submitted, 2007.

[42] T. Odijk. Macromolecules, 29:1842, 1996. 